\title{
Buried Object Detection by an Inexact Newton Method Applied to Nonlinear Inverse Scattering
}

\author{
Matteo Pastorino and Andrea Randazzo \\ Department of Naval, Electrical, Electronic, and Telecommunication Engineering, University of Genoa, Via Opera Pia 11A, \\ 16145 Genova, Italy \\ Correspondence should be addressed to Matteo Pastorino, matteo.pastorino@unige.it
}

Received 20 March 2012; Revised 11 June 2012; Accepted 12 June 2012

Academic Editor: Kristen M. Donnell

Copyright ( 2012 M. Pastorino and A. Randazzo. This is an open access article distributed under the Creative Commons Attribution License, which permits unrestricted use, distribution, and reproduction in any medium, provided the original work is properly cited.

\begin{abstract}
An approach to reconstruct buried objects is proposed. It is based on the integral equations of the electromagnetic inverse scattering problem, written in terms of the Green's function for half-space geometries. The full nonlinearity of the problem is exploited in order to inspect strong scatterers. After discretization of the continuous model, the resulting equations are solved in a regularization sense by means of a two-step inexact Newton algorithm. The capabilities and limitations of the method are evaluated by means of some numerical simulations.
\end{abstract}

\section{Introduction}

Subsurface imaging is an important subject in several applicative areas, including seismic and geophysical prospecting, nondestructive, testing and medical diagnostics [139]. Electromagnetic techniques are widely applied to face this problem. Ground penetrating radar [40] is the basic instrumentation able to retrieve discontinuities in the lower subspace (the ground). Processing of GPR data can allow an improvement in the detection and localization capabilities of the system. In some cases, neural network-based approaches have been adopted [41]. Moreover, inverse-scattering-based techniques have been proposed, too. By using these methods, the measured values of the field scattered by the discontinuities present in the lower half space are "inverted" in order to obtain the spatial distributions of dielectric parameters (e.g., the dielectric permittivity and the electric conductivity) inside a fixed inspection domain. Approaches of this kind have been proposed, for example, for the detection of water leaking from pipes [42]. If an accurate model of the buried scatterers is available, it would be possible to retrieve information about the buried objects in terms of their dielectric parameters.
The present authors developed in [43] an inversescattering-based method for buried object imaging under the second-order Born approximation (SOBA) [21, 44-48]. By using the SOBA, the reconstruction of buried objects has been obtained with a better accuracy with respect to linearized approximations. In particular, due to the nonlinear nature of the scattering equations, in which the electric field inside the inspected region is an unknown quantity as well as the spatial distributions of the dielectric properties of the medium, the SOBA allows to approximately retrieve the field distribution, too.

However, in order to detect strong discontinuities, the exact nonlinear inverse scattering formulation must be considered. This approach has been faced in $[49,50]$, in which strong scatterers have been reconstructed in free space under tomographic imaging conditions. The approach proposed in [49], which is based on the use an inexact Newton method, has been experimentally tested in [51]. The same method has been successfully applied in [52] within the contrast source formulation (under free-space conditions).

In the present paper, the approach developed in [43] for buried object detection under the SOBA is extended to treat strong scatterers. In this case, the exact nonlinear inverse 


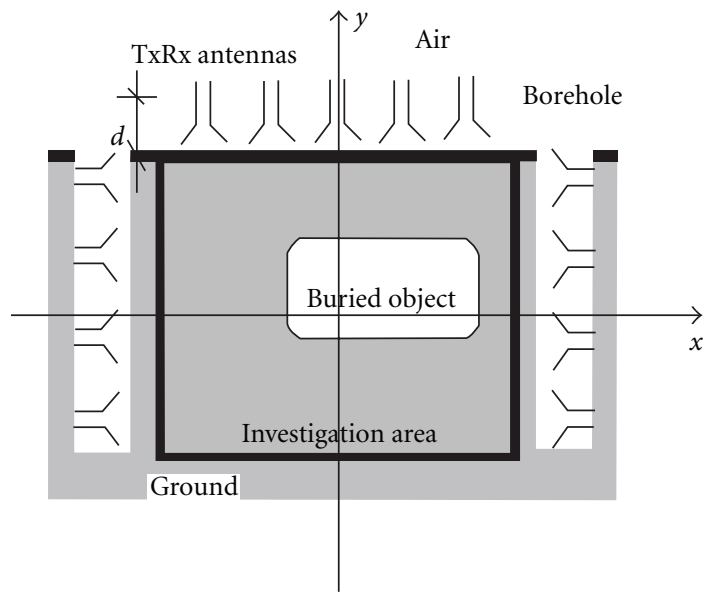

FIgURE 1: Geometrical configuration of the problem.

scattering formulation, in terms of the half-space Green's function, is taken into account. In particular, starting from the integral equations for the scattered field for cylindrical objects, slides of the spatial distributions of the dielectric parameters are reconstructed by numerically solving these equations in a regularization sense.

The paper is organized as follows. In Section 2 the mathematical formulation of the approach is reported. In Section 3, the application of the inexact Newton method is described. Numerical results are reported in Section 4, and conclusions are drawn in Section 5.

\section{Mathematical Formulation}

Let us consider a cylindrical object buried in a homogeneoushalf-space medium (Figure 1). A 2D transverse-magnetic (TM) configuration is assumed. The dielectric properties of the buried object are assumed to be independent of the $z$ coordinate. The transmitting and receiving antennas are located in the transverse plane and generate a $z$-polarized electric field in both the half spaces (this field is independent of the axial coordinate, too).

The dielectric characteristics of the investigation domain $S$, in the lower half space, are described by the so-called object function, which is defined as

$$
\tau(\mathbf{r})=j \omega\left[\epsilon(\mathbf{r})-\epsilon_{b}\right]
$$

where $\epsilon(\mathbf{r})$ is the complex dielectric permittivity at point $\mathbf{r}$, and $\epsilon_{b}$ denotes the dielectric permittivity of the soil.

The investigation area is illuminated by $V$ line-current sources, with angular frequency $\omega$ (in the following, the $e^{j \omega t}$ time dependence is assumed and omitted). A mixed illumination configuration (partially borehole and partially from the top), which allows direct comparisons with the results reported in [43], is exploited. Under such assumptions, also the scattered electric field is $z$-polarized and independent of $z$. Consequently, only the transverse plane can be considered for the imaging procedure.
The scattered electric field, $u_{\text {scatt }}^{(v)}, v=1, \ldots, V$, is collected for any incident wave in $M$ measurement points $\mathbf{s}_{m}^{(v)}$, which constitute the so-called observation domain $O$. The dielectric properties of $S$ can be related to the scattered field by means of the following set of nonlinear integral equations:

$$
\begin{gathered}
u_{\text {scatt }}^{(v)}(\mathbf{r})=G_{\text {lower }}^{\text {upper }} \tau u_{\text {tot }}^{(v)}(\mathbf{r}), \quad \mathbf{r} \in O, \\
u_{\text {tot }}^{(v)}(\mathbf{r})=u_{\text {inc }}^{(v)}(\mathbf{r})+G_{\text {lower }}^{\text {lower }} \tau u_{\text {tot }}^{(v)}(\mathbf{r}), \quad \mathbf{r} \in S,
\end{gathered}
$$

where $G_{\text {lower }}^{\text {upper }}(\cdot)(\mathbf{r})=j \omega \mu_{0} \int_{S}(\cdot) g_{\mathrm{HS}}\left(\mathbf{r}, \mathbf{r}^{\prime}\right) d \mathbf{r}^{\prime}, \mathbf{r} \in O$ and $G_{\text {lower }}^{\text {lower }}(\cdot)(\mathbf{r})=j \omega \mu_{0} \int_{S}(\cdot) g_{\mathrm{HS}}\left(\mathbf{r}, \mathbf{r}^{\prime}\right) d \mathbf{r}^{\prime}, \mathbf{r} \in S$ are the data and state operators, where $g_{\mathrm{HS}}$ is the half-space Green's function [43] (superscripts in the operators recall where they are calculated, whereas subscripts recall where they are integrated).

Equations (2) can be rewritten in a compact operator form as

$$
\operatorname{HS}(\mathbf{x})=\mathbf{y},
$$

where $\mathbf{x}=\left[\begin{array}{llll}\tau & u_{\mathrm{tot}}^{(1)} & \cdots & u_{\mathrm{tot}}^{(V)}\end{array}\right]^{t}$ is an array containing the values of the object function and of the total electric field inside the investigation area for any illumination. In (3), y =

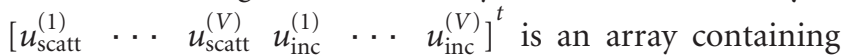
the values of the scattered electric field acquired by the measurement probes in the upper half space and the values of the incident electric field inside the investigation area. The half-space operator HS can be expressed, according to (2), as

$$
\mathrm{HS}(\mathbf{x})=\left[\begin{array}{c}
G_{\text {lower }}^{\text {upper }} \tau u_{\text {tot }}^{(1)} \\
\vdots \\
G_{\text {lower }}^{\text {upper }} \tau u_{\text {tot }}^{(V)} \\
u_{\text {tot }}^{(1)}-G_{\text {lower }}^{\text {lower }} \tau u_{\text {tot }}^{(1)} \\
\vdots \\
u_{\text {tot }}^{(V)}-G_{\text {lower }}^{\text {lower }} \tau u_{\text {tot }}^{(V)}
\end{array}\right] .
$$

As it is well known, the inverse problem (4) is non-linear and ill-posed. Consequently, nonlinear regularizing methods must be used.

\section{Regularized Solution of $(3)$}

Due to the mentioned ill posedness, (3) must be solved in a regularized sense. The inexact Newton regularizing scheme developed in $[49,50]$ is applied here. This method includes two nested loops, in which a linearization (with respect to the current solution guess $\mathbf{x}_{k}$ ) of the operator HS is constructed (outer loop) and solved by means of the truncated Landweber method (inner loop). Exactly as in [49], the algorithm performs the following steps.

(1) For $k=0$ ( $k$ denotes the outer iteration index), a starting guess $\mathbf{x}_{0}$ is chosen (initialization phase).

(2) The following linearized version of equation is constructed

$$
\mathrm{HS}_{\mathbf{x}_{k}}^{\prime} \mathbf{h}=\mathbf{y}_{k} \text {, }
$$


where $\mathbf{h}=\left[\begin{array}{lll}h_{\tau} & h_{u}^{(1)} \cdots & h_{u}^{(V)}\end{array}\right]^{t}, \mathbf{y}_{k}=\mathbf{y}-\operatorname{HS}\left(\mathbf{x}_{k}\right)$, and $\mathrm{HS}_{\mathbf{x}_{k}}^{\prime}$ is the Fréchet derivative of $\mathrm{HS}$ at point $\mathbf{x}_{k}$. A regularized solution $\hat{\mathbf{h}}_{k}$ to (5) is obtained by applying an iterative truncated Landweber algorithm, for which we have

$$
\begin{gathered}
\mathbf{h}_{k, 0}=0, \\
\mathbf{h}_{k, l+1}=\mathbf{h}_{k, l}-\gamma \mathrm{HS}_{\mathbf{x}_{k}}^{\prime} *\left(H S_{\mathbf{x}_{k}}^{\prime} \mathbf{h}_{k, l}-\mathbf{y}_{k}\right),
\end{gathered}
$$

where $\mathrm{HS}_{\mathbf{x}_{k}}^{\prime}{ }^{*}$ is the adjoint operator of $\mathrm{HS}_{\mathbf{x}_{k}}^{\prime}$.

(3) The current solution is updated by using the following relation:

$$
\mathbf{x}_{k+1}=\mathbf{x}_{k}+\hat{\mathbf{h}}_{k}
$$

(4) A predefined stopping rule is finally applied. If this criterion is not satisfied, a fixed maximum number of outer iterations, $k_{\max }$, is performed. In both cases, at any iteration, the procedure can be stopped, otherwise steps (2)-(4) are repeated.

It should be noted that step (2) is actually the inner loop of the algorithm, whereas steps (1)-(3) constitute the outer loop.

\section{Numerical Results}

The developed method has been tested by means of numerical simulations. The working frequency has been set equal to $300 \mathrm{MHz}$ (i.e., similar to that used in ground penetrating radars). A homogeneous lossy soil characterized by a relative dielectric permittivity $\epsilon_{r_{b}}=4$ and an electric conductivity $\sigma_{b}=0.01 \mathrm{~S} / \mathrm{m}$ has been considered (in order to simulate a dry sandy soil [53]). Similar to [43], a mixed measurement configuration is assumed, where reflection and crossborehole operating modes are mixed together in order to acquire as much information as possible. Correspondingly, the antennas are located at points (Cartesian coordinates)

$$
\mathbf{r}_{n}^{\mathrm{ant}}= \begin{cases}\left(-\frac{2}{3} \lambda_{0},-\frac{4}{3} \lambda_{0}+(n-1) \frac{2 \lambda_{0}}{21}\right), & n=1, \ldots, 15 \\ \left(-\frac{2}{3} \lambda_{0}+(n-15) \frac{2 \lambda_{0}}{21}, 0\right), & n=16, \ldots, 28 \\ \left(\frac{2}{3} \lambda_{0},-(n-29) \frac{2 \lambda_{0}}{21}\right), & n=29, \ldots, 43\end{cases}
$$

where $\lambda_{0}$ is the free-space wavelength in the upper medium. A subset of these antennas act both as transmitter (TX) and receiver $(\mathrm{RX})$. When an antenna operates in TX mode, all the remaining ones collect the scattered electric field (i.e., they work in RX mode). Consequently, for every illumination, $M=42$ field samples are collected. In particular, in the cases reported in the following, $V=7$ antennas are used. They are located at positions denoted by the indexes given by $n=7(v-1)+1, v=1, \ldots, V$.
The investigation area is a square domain of dimensions $\lambda_{0} \times \lambda_{0}$, whose upper side coincides with the airground interface. In order to numerically solve the equations involved in the inverse-scattering problem, such domain has been discretized into $N=20 \times 20$ square subdomains.

The scattered field values used as input data for the considered simulations have been numerically obtained by using a computational code based on the method of moments [54]. In order to avoid the so-called inverse crime, a finer discretization (such as the ratio of the cell dimensions in the direct and inverse problems is not an integer number) has been used for the computation of the synthetic data. Moreover, in order to simulate a more realistic scenario, a Gaussian noise with zero mean value and variance corresponding to a signal-to-noise ratio $\mathrm{SNR}=25 \mathrm{~dB}$ has also been added to the computed data.

In all the simulations reported in this section, the initialization phase has been always performed by assuming an empty investigation domain in the lower half space. Consequently, in this phase, the object function is equal to zero, and the total electric field is assumed equal to the one transmitted in the lower half space.

The first reported case concerns the reconstruction of a single circular cylinder of radius $a=0.2 \lambda_{0}$ centered at $\mathbf{r}^{\mathrm{obj}}=$ $\left(0.2 \lambda_{0},-0.5 \lambda_{0}\right)$. The dielectric properties of the cylinder are $\epsilon_{r_{\mathrm{obj}}}=5.6$ and $\sigma_{\mathrm{obj}}=0.01 \mathrm{~S} / \mathrm{m}$. The parameters of the inexact Newton algorithm are the following: maximum number of inner iterations, $k_{\mathrm{LW}_{\max }}=30$; maximum number of outer iterations, $k_{\mathrm{IN}_{\max }}=100$. Figure 2 reports the behavior of two error parameters for different values of the number of outer iterations of the iterative procedure. In particular, the two parameters are defined as

$$
\begin{gathered}
r_{\text {data }}=\frac{\left\|u^{\text {actual }}-u^{\text {calc }}\right\|_{2}}{\left\|u^{\text {actual }}\right\|_{2}}, \\
r_{\text {solution }}=\frac{\left\|\tau^{\text {actual }}-\tau^{\text {rec }}\right\|_{2}}{\left\|\tau^{\text {actual }}\right\|_{2}},
\end{gathered}
$$

with $\tau^{\text {actual }}$ and $\tau^{\text {rec }}$ being the actual and reconstructed permittivity distributions (at the current iteration) and $u^{\text {actual }}$ and $u^{\text {calc }}$ the measured and computed values of the electric field data (at the current iteration, too). As can be seen from Figure 2, the plot of the "residual" on the data (the error parameter $\left.r_{\text {data }}\right)$ decreases monotonically as expected. Moreover, the plot of the solution "residual" (the error parameter $r_{\text {solution }}$ ) shows a typical semiconvergence behavior, that is, after a certain value of the iteration number the reconstruction quality degrades $[55,56]$.

The final image of the distribution of the relative dielectric permittivity inside the investigation region (corresponding to the optimum iteration number) is shown in Figure 3. As can be seen, the method is able to correctly retrieve the dielectric profile of the investigation area. In particular, both the shape of the buried inclusion and the value of its dielectric permittivity are correctly identified. 


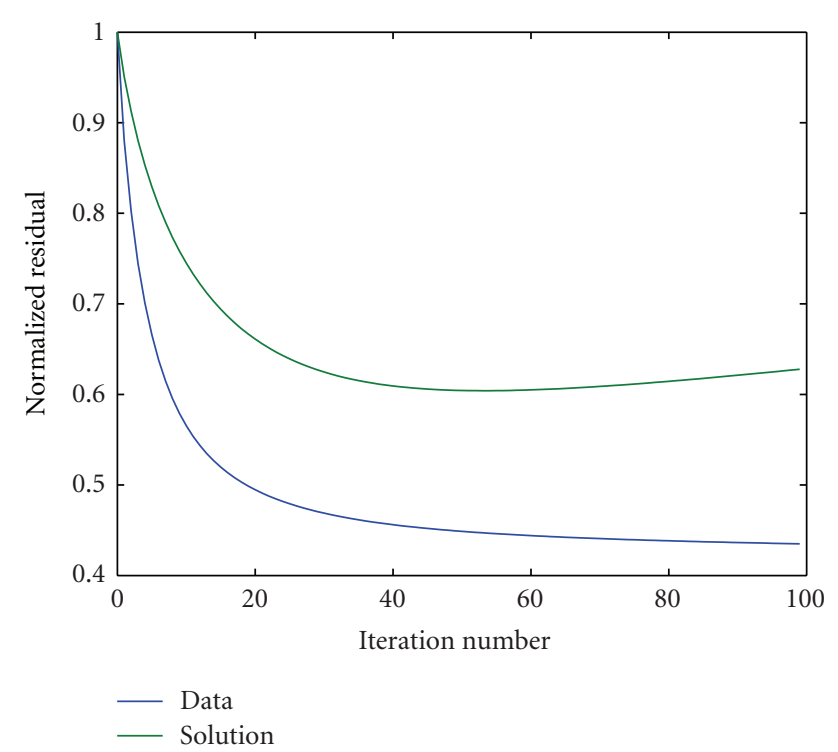

Figure 2: Data and solution residuals versus the outer iteration number (error parameters of (9)). Single circular cylinder buried in a lossy half space.

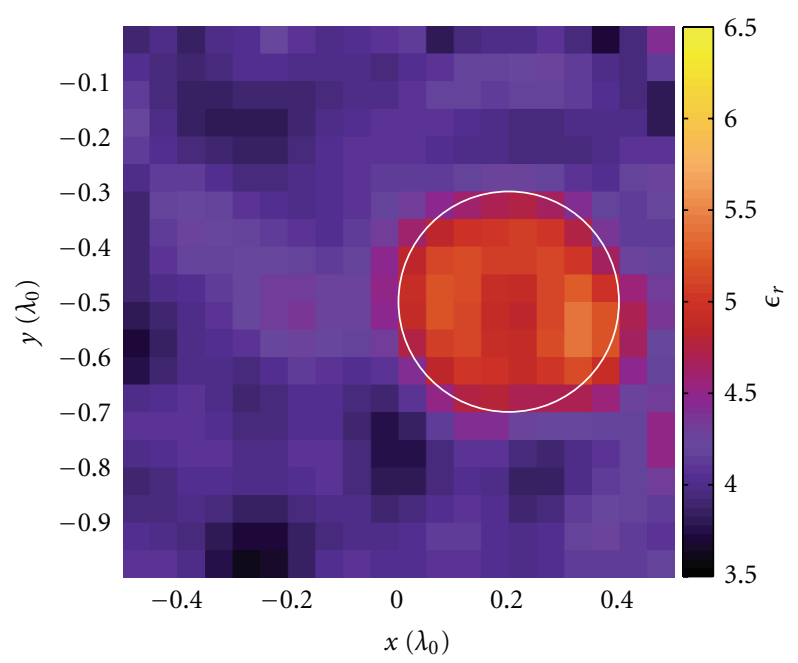

FIGURE 3: Reconstructed distribution of the relative dielectric permittivity. Single circular cylinder buried in a lossy half space.

For the present case, an analysis of the effects of the noise has also been performed. In particular, the mean relative error on the reconstruction, defined as the $L_{1}$-norm

$$
e=\frac{1}{N} \sum_{n=1}^{N} \frac{\left|\epsilon_{n}^{\text {actual }}-\epsilon_{n}^{\text {rec }}\right|}{\left|\epsilon_{n}^{\text {actual }}\right|}
$$

has been calculated. In (10), $\epsilon_{n}^{\text {actual }}$ and $\epsilon_{n}^{\text {rec }}$ denote the values of the original and reconstructed complex permittivity inside the $n$th discretization subdomain of the investigation region. Moreover, the optimal value of the outer iteration have been evaluated, too. The obtained results are reported in Table 1.

As expected, as the noise level increases, the reconstruction "quality" decreases. However, even in presence of strong
TABLE 1: Mean relative errors and optimal iteration numbers versus the signal-to-noise ratio. Single circular cylinder buried in a lossy half space.

\begin{tabular}{lcccc}
\hline SNR & 25 & 20 & 15 & 10 \\
Mean relative error & 0.065 & 0.078 & 0.085 & 0.12 \\
Optimum outer iteration number & 53 & 34 & 14 & 5 \\
\hline
\end{tabular}

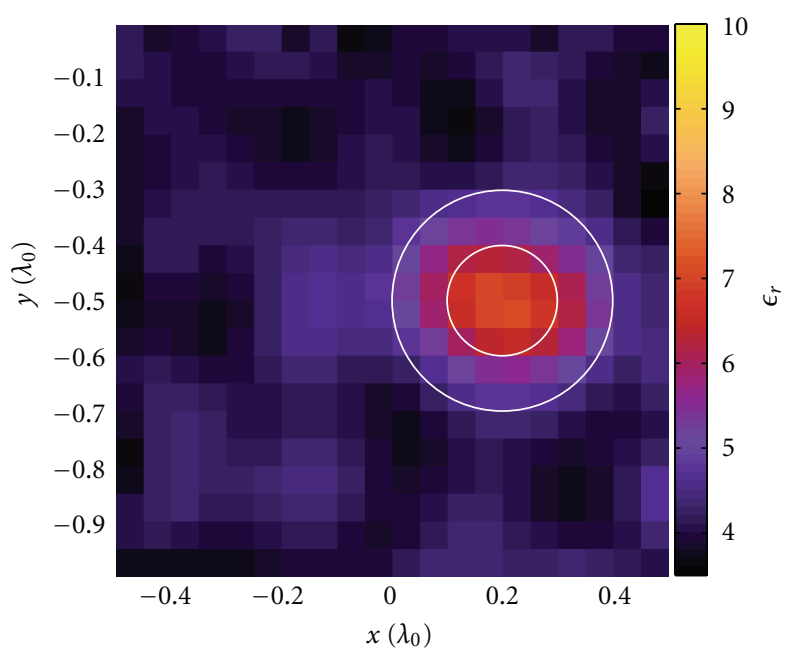

FIgURE 4: Reconstructed distribution of the relative dielectric permittivity. Double-layer circular cylinder buried in a lossy half space.

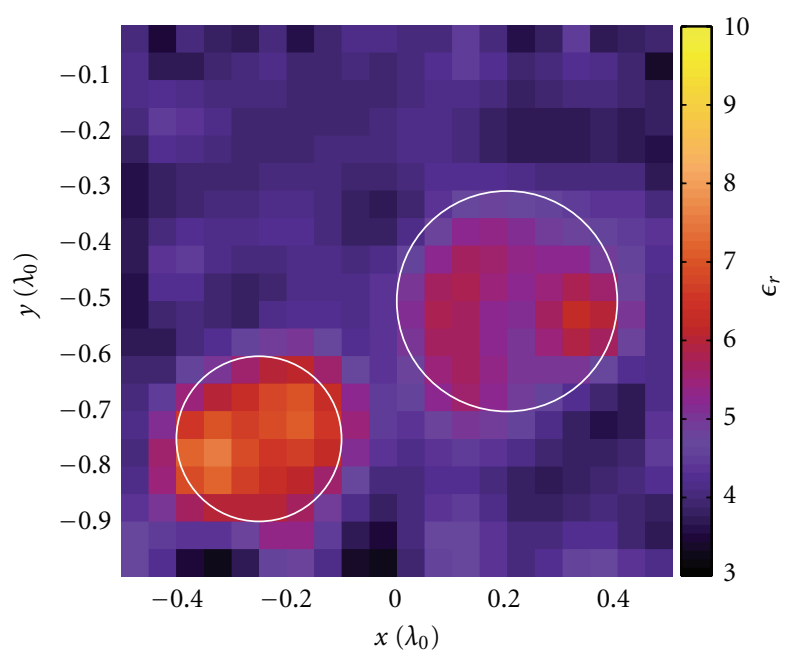

Figure 5: Reconstructed distribution of the relative dielectric permittivity. Two separate circular cylinders buried in a lossy half space.

noise levels, the method is able to yield rather good reconstructions (e.g., the error parameter $e$ is equal to about $8 \%$ error for $\mathrm{SNR}=15 \mathrm{~dB}$ ).

In the second set of simulations, the reconstruction of a more complex configuration has been considered. In particular, the presence of a double-layer circular cylinder has been assumed. The outer layer has radius $a_{1}=0.2 \lambda_{0}$ and is characterized by a relative dielectric permittivity $\epsilon_{r_{1}}=$ 5.6 and an electric conductivity $\sigma_{1}=0.01 \mathrm{~S} / \mathrm{m}$, whereas 


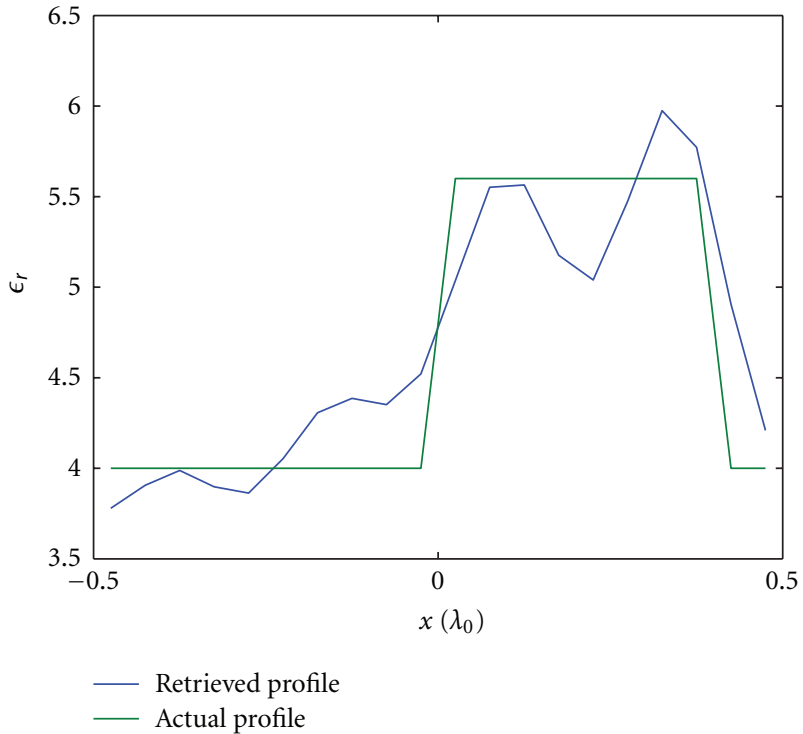

(a)

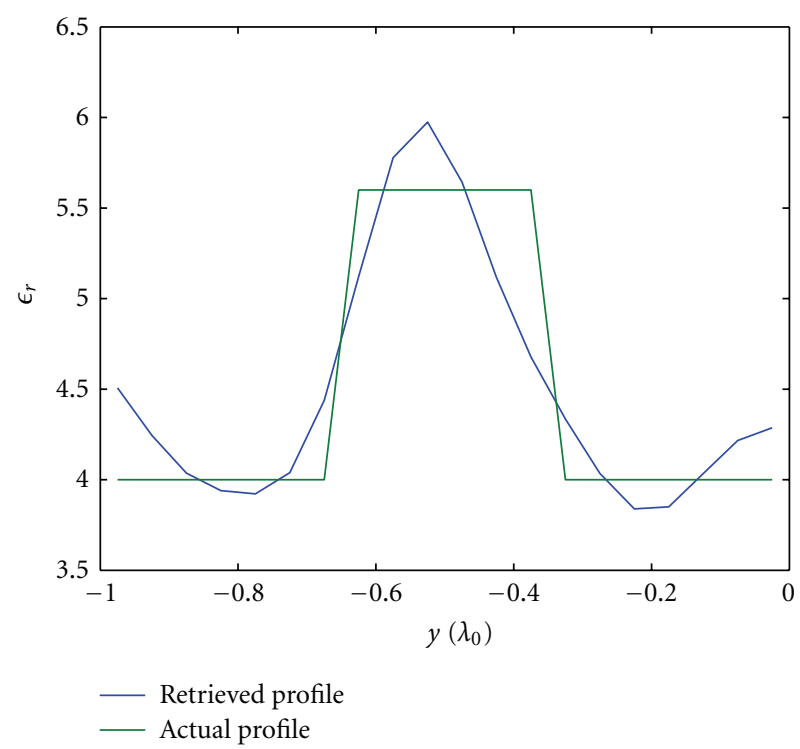

(c)

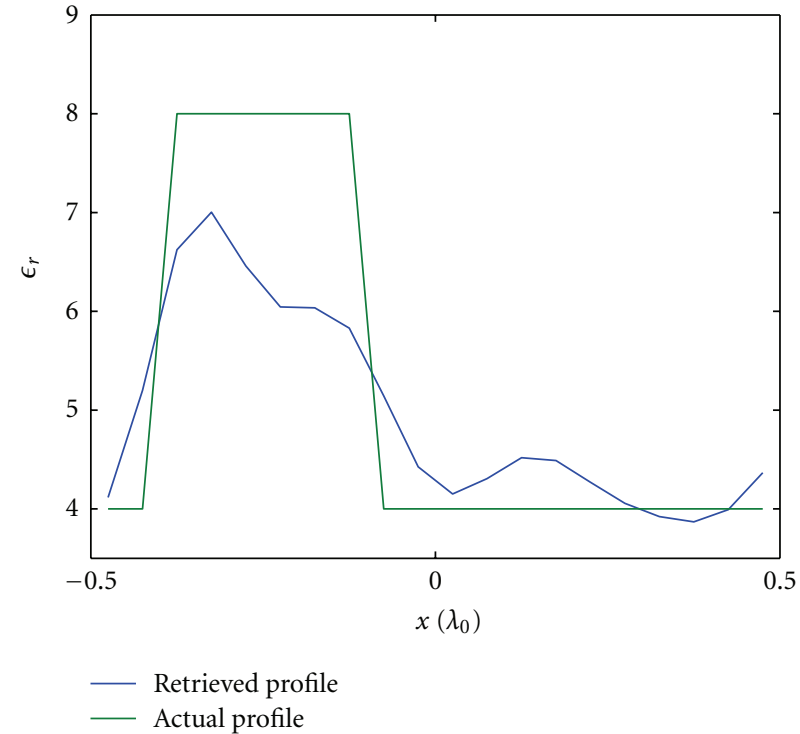

(b)

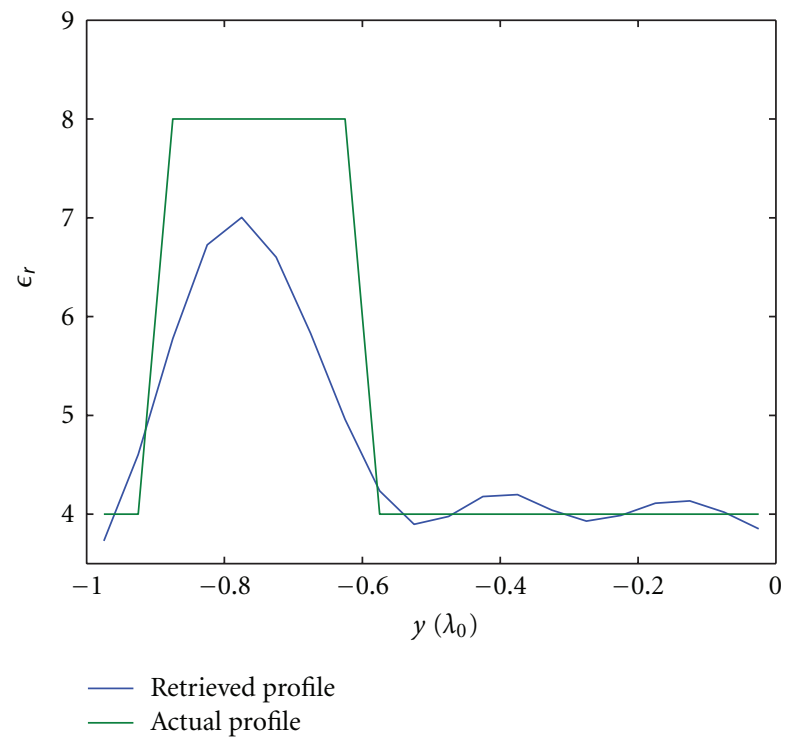

(d)

Figure 6: Horizontal cuts ((a) $y=-0.525 \lambda_{0}$ and (b) $\left.y=-0.775 \lambda_{0}\right)$ ) and vertical cuts ((c) $x=0.325 \lambda_{0}$ and (d) $\left.\left.x=-0.325 \lambda_{0}\right)\right)$ of the reconstructed distribution of the relative dielectric permittivity (Figure 4). Two circular cylinders buried in a lossy half space.

the inner one has a radius $a_{2}=0.1 \lambda_{0}$ and is characterized by a relative dielectric permittivity $\epsilon_{r_{2}}=8$ and an electric conductivity $\sigma_{2}=0.01 \mathrm{~S} / \mathrm{m}$. The object is centered at $\mathbf{r}^{\mathrm{obj}}=\left(0.2 \lambda_{0},-0.5 \lambda_{0}\right)$. The parameters of the inexact Newton method are the same as the ones adopted in the first case. The reconstructed distribution of the relative dielectric permittivity (final image) is shown in Figure 4. As can be seen, the object is correctly located, and the values of the dielectric properties are identified with a quite good accuracy.

Finally, the reconstruction of two separate circular cylinders have been considered. The radii of the two cylinders are $a_{1}=0.2 \lambda_{0}$ and $a_{2}=0.15 \lambda_{0}$. They are buried with centers located at points $\mathbf{r}_{1}^{\mathrm{obj}}=\left(0.2 \lambda_{0},-0.5 \lambda_{0}\right)$ and $\mathbf{r}_{2}^{\mathrm{obj}}=$ $\left(-0.25 \lambda_{0},-0.75 \lambda_{0}\right)$. The dielectric properties of the two scatterers are the following: $\epsilon_{r_{1}}=5.6, \epsilon_{r_{2}}=8$, and $\sigma_{1}=\sigma_{2}=0.01 \mathrm{~S} / \mathrm{m}$. The signal-to-noise ratio is SNR $=$ $25 \mathrm{~dB}$. The parameters of the iterative inversion algorithm are the same as in the previous cases. The reconstructed distribution of the relative dielectric permittivity is shown in Figure 5. This image corresponds to the optimal solution. As can be seen, also in this quite complex case, the method is able to correctly reconstruct the investigated scene. In particular, the two objects are clearly identified and separated. Moreover, both shapes and dielectric properties of the two targets are estimated with quite good accuracies 
(although for the second cylinder the dielectric permittivity is slightly underestimated). In order to better assess the reconstruction accuracy, Figure 6 gives the profiles of the retrieved distribution of Figure 5 along some cuts parallel to the coordinate axes.

\section{Conclusion}

In this paper, a previously proposed approach for the imaging of buried objects has been extended to inspect strong scatterers, for which the exact nonlinear equations of the inverse scattering problem must be considered. The developed method is based on a two-step inexact Newton method, which allows us to obtain a regularized solution of the (discretized) integral equations relating the samples of scattered electric field collected in the upper half space to the distributions of the dielectric parameters inside the test domain located in the ground. The reported preliminary results of numerical simulations seem to indicate the validity of the proposed approach, which needs further evaluation assessments, including an experimental validation, which will be the subject of future research activity in this area.

\section{References}

[1] T. J. Cui and W.C. Chew, "Inverse-scattering methods for three-dimensional targets buried in the lossy earth," in Proceedings of the IEEE International Symposium on Antennas and Propagation, vol. 3, pp. 1776-1779, 2000.

[2] C. J. Lin and C. C. Chiu, "Inverse scattering of buried inhomogeneous dielectric material coated on a conductor," in Proceedings of the 5th International Kharkov Symposium on Physics and Engineering of Microwaves, Millimeter, and Submillimeter Waves (MSMW'04), pp. 281-283, June 2004.

[3] R. Persico, R. Bernini, and F. Soldovieri, "The role of the measurement configuration in inverse scattering from buried objects under the born approximation," IEEE Transactions on Antennas and Propagation, vol. 53, no. 6, pp. 1875-1887, 2005.

[4] C. J. Lin and C. C. Chiu, "Inverse scattering of buried complex object by TE wave illumination," in Proceedings of the IEEE International Symposium on Microwave, Antenna, Propagation and EMC Technologies for Wireless Communications (MAPE '05), pp. 784-787, August 2005.

[5] A. N. Askarpour and R. Faraji-Dana, "Inverse scattering of 2$\mathrm{D}$ dielectric objects buried in multilayer media using complex images green's function in born iteration method," in Proceedings of the IEEE Antennas and Propagation Society International Symposium (APS '06), pp. 1085-1088, July 2006.

[6] C. C. Chiu and Y. W. Kiang, "Electromagnetic inverse scattering of a conducting cylinder buried in a lossy half-space," IEEE Transactions on Antennas and Propagation, vol. 40, no. 12, pp. 1562-1563, 1992.

[7] X. Chen, K. R. Shao, Y. Guo, J. Zhu, and J. D. Lavers, "A inverse scattering technique for objects buried in planar layered media based on an estimation of distribution algorithm," in Proceedings of the 14th Biennial IEEE Conference on Electromagnetic Field Computation (CEFC'10), May 2010.

[8] L. Crocco, M. D’Urso, and T. Isernia, "New models and tools for forward and inverse scattering from buried dielectric targets," in Proceedings of the 10th International Conference Ground Penetrating Radar (GPR '04), pp. 59-62, June 2004.
[9] K. Belkebir, R. E. Kleinman, and C. Pichot, "Microwave imaging-location and shape reconstruction from multifrequency scattering data," IEEE Transactions on Microwave Theory and Techniques, vol. 45, no. 4, pp. 469-476, 1997.

[10] L. Xu, S. K. Davis, S. C. Hagness, D. W. van der Weide, and B. D. Van Veen, "Microwave imaging via space-time beamforming: experimental investigation of tumor detection in multilayer breast phantoms," IEEE Transactions on Microwave Theory and Techniques, vol. 52, no. 8, pp. 1856-1865, 2004.

[11] T. J. Cui, A. A. Aydiner, W. C. Chew, D. L. Wright, and D. V. Smith, "Three-dimensional imaging of buried objects in very lossy earth by inversion of VETEM data," IEEE Transactions on Geoscience and Remote Sensing, vol. 41, no. 10, pp. 2197-2210, 2003.

[12] M. Lambert and D. Lesselier, "Binary-constrained inversion of a buried cylindrical obstacle from complete and phaseless magnetic fields," Inverse Problems, vol. 16, no. 3, pp. 563-576, 2000.

[13] G. C. Giakos, M. Pastorino, F. Russo, S. Chiwdhury, N. Shah, and W. Davros, "Noninvasive imaging for the new century," IEEE Instrumentation \& Measurement Magazine, vol. 2, pp. 32-35, 1999.

[14] R. Zoughi, Microwave Nondestructive Testing and Evaluation, Kluwer Academic, Amsterdam, The Netherlands, 2000.

[15] D. Lesselier and J. Bowler, "Foreword to the special section on electromagnetic and ultrasonic nondestructive evaluation," Inverse Problems, vol. 18, no. 6, pp. 1-2, 2002.

[16] S. Kharkovsky and R. Zoughi, "Microwave and millimeter wave nondestructive testing and evaluation," IEEE Instrumentation and Measurement Magazine, vol. 10, no. 2, pp. 26-38, 2007.

[17] C. C. Chen, J. T. Johnson, M. Sato, and A. G. Yarovoy, "Guest editorial foreword to the special issue on subsurface sensing using Ground-Penetrating Radar (GPR)," IEEE Transactions on Geoscience and Remote Sensing, vol. 45, no. 8, p. 2419, 2007.

[18] O. Dorn and D. Lesselier, "Special issue on electromagnetic inverse problems: emerging methods and novel applications," Inverse Problems, vol. 26, no. 7, 2010.

[19] A. Baussard, E. L. Miller, and D. Lesselier, "Adaptive multiscale reconstruction of buried objects," Inverse Problems, vol. 20, no. 6, supplement, pp. S1-S15, 2004.

[20] A. Massa, M. Pastorino, and A. Randazzo, "Reconstruction of two-dimensional buried objects by a differential evolution method," Inverse Problems, vol. 20, no. 6, supplement, pp. S135-S150, 2004.

[21] M. Pastorino, Microwave Imaging, John Wiley \& Sons, Hoboken, NJ, USA, 2010.

[22] C. H. Kuo and M. Moghaddam, "Electromagnetic scattering from a buried cylinder in layered media with rough interfaces," IEEE Transactions on Antennas and Propagation, vol. 54, no. 8, pp. 2392-2401, 2006.

[23] A. Bréard, G. Perrusson, and D. Lesselier, "Hybrid differential evolution and retrieval of buried spheres in subsoil," IEEE Geoscience and Remote Sensing Letters, vol. 5, pp. 788-792, 2008.

[24] A. Tabatabaeenejad and M. Moghaddam, "Inversion of subsurface properties of layered dielectric structures with random slightly rough interfaces using the method of simulated annealing," IEEE Transactions on Geoscience and Remote Sensing, vol. 47, no. 7, pp. 2035-2046, 2009.

[25] M. El-Shenawee, O. Dorn, and M. Moscoso, "An adjoint-field technique for shape reconstruction of 3-D penetrable object immersed in lossy medium," IEEE Transactions on Antennas and Propagation, vol. 57, no. 2, pp. 520-534, 2009. 
[26] P. M. Meaney, M. W. Fanning, D. Li, S. P. Poplack, and K. D. Paulsen, "A clinical prototype for active microwave imaging of the breast," IEEE Transactions on Microwave Theory and Techniques, vol. 48, no. 1, pp. 1841-1853, 2000.

[27] G. Bozza, M. Brignone, and M. Pastorino, "Application of the no-sampling linear sampling method to breast cancer detection," IEEE Transactions on Biomedical Engineering, vol. 57, no. 10, pp. 2525-2534, 2010.

[28] G. Bellizzi, O. Bucci, and I. Catapano, "Microwave cancer imaging exploiting magnetic nanoparticles as contrast agent," IEEE Transactions on Biomedical Engineering, vol. 58, pp. 2528-2536, 2011.

[29] H. Harada, D. J. N. Wall, T. Takenaka, and M. Tanaka, "Conjugate gradient method applied to inverse scattering problem," IEEE Transactions on Antennas and Propagation, vol. 43, no. 8, pp. 784-792, 1995.

[30] P. M. van den Berg and A. Abubakar, "Contrast source inversion method: state of art," Journal of Electromagnetic Waves and Applications, vol. 15, no. 11, pp. 1503-1505, 2001.

[31] I. T. Rekanos, "Shape reconstruction of a perfectly conducting scatterer using differential evolution and particle swarm optimization," IEEE Transactions on Geoscience and Remote Sensing, vol. 46, no. 7, pp. 1967-1974, 2008.

[32] A. Litman, D. Lesselier, and F. Santosa, "Reconstruction of a two-dimensional binary obstacle by controlled evolution of a level-set," Inverse Problems, vol. 14, no. 3, pp. 685-706, 1998.

[33] R. Ferrayé, J. Y. Dauvignac, and C. Pichot, "Reconstruction of complex and multiple shape object contours using a level set method," Journal of Electromagnetic Waves and Applications, vol. 17, no. 2, pp. 153-181, 2003.

[34] A. Abubakar and T. M. Habashy, "Constrained parametric minimization for the inversion of electromagnetic measurements in the earth," in Proceedings of the Progress in Electromagnetics Research Symposium (PIERS '04), pp. 49-52, March 2004.

[35] D. Colton, H. Haddar, and M. Piana, "The linear sampling method in inverse electromagnetic scattering theory," Inverse Problems, vol. 19, no. 6, supplement, pp. S105-S137, 2003.

[36] L. Pan, X. Chen, Y. Zhong, and S. P. Yeo, "Comparison among the variants of subspace-based optimization method for addressing inverse scattering problems: transverse electric case," Journal of the Optical Society of America A, vol. 27, no. 10, pp. 2208-2215, 2010.

[37] Y. Zhong, X. Chen, and K. Agarwal, "An improved subspacebased optimization method and its implementation in solving three-dimensional inverse problems," IEEE Transactions on Geoscience and Remote Sensing, vol. 48, no. 10, pp. 3763-3768, 2010.

[38] J. De Zaeytijd, A. Franchois, C. Eyraud, and J. M. Geffrin, "Full-wave three-dimensional microwave imaging with a regularized Gauss-Newton method-theory and experiment," IEEE Transactions on Antennas and Propagation, vol. 55, no. 11, pp. 3279-3292, 2007.

[39] C. Gilmore, P. Mojabi, and J. LoVetri, "Comparison of an enhanced distorted born iterative method and the multiplicative-regularized contrast source inversion method," IEEE Transactions on Antennas and Propagation, vol. 57, no. 8, pp. 2341-2351, 2009.

[40] D. J. Daniels, Ground Penetrating Radars, IEE, London, UK, 2nd edition, 2004.

[41] R. Mittra, M. Ji-Fu, and Y. Wenhua, "Identifying buried objects using the neural network approach," in Proceedings of the IEEE Antennas and Propagation Society International Symposium, pp. 2596-2599, 1999.
[42] L. Crocco, G. Prisco, F. Soldovieri, and N. J. Cassidy, "Advanced forward modeling and tomographic inversion for leaking water pipes monitoring," in Proceedings of the 4th International Workshop on Advanced Ground Penetrating Radar (IWAGPR '07), pp. 127-131, June 2007.

[43] G. Bozza, C. Estatico, M. Pastorino, and A. Randazzo, "Application of an inexact-Newton method within the second-order Born approximation to buried objects," IEEE Geoscience and Remote Sensing Letters, vol. 4, no. 1, pp. 51-55, 2007.

[44] R. Pierri and G. Leone, "Inverse scattering of dielectric cylinders by a second-order born approximation," IEEE Transactions on Geoscience and Remote Sensing, vol. 37, no. 1, pp. 374$382,1999$.

[45] A. Brancaccio, V. Pascazio, and R. Pierri, "Quadratic model for inverse profiling: the one-dimensional case," Journal of Electromagnetic Waves and Applications, vol. 9, no. 5-6, pp. 673-696, 1995.

[46] C. Estatico, M. Pastorino, and A. Randazzo, "An inexactNewton method for short-range microwave imaging within the second-order born approximation," IEEE Transactions on Geoscience and Remote Sensing, vol. 43, no. 11, pp. 2593-2605, 2005.

[47] S. Caorsi, A. Costa, and M. Pastorino, "Microwave imaging within the second-order born approximation: stochastic optimization by a genetic algorithm," IEEE Transactions on Antennas and Propagation, vol. 49, no. 1, pp. 22-31, 2001.

[48] G. Bozza, C. Estatico, M. Pastorino, and A. Randazzo, "A regularization approach to microwave imaging under the secondorder Born approximation with real data," in Proceedings of the IEEE International Workshop on Imaging Systems and Techniques, Niagara Falls, pp. 14-19, Ontario, Canada, May 2005.

[49] G. Bozza, C. Estatico, M. Pastorino, and A. Randazzo, "An inexact Newton method for microwave reconstruction of strong scatterers," IEEE Antennas and Wireless Propagation Letters, vol. 5, no. 1, pp. 61-64, 2006.

[50] G. Bozza, C. Estatico, A. Massa, M. Pastorino, and A. Randazzo, "Short-range image-based method for the inspection of strong scatterers using microwaves," IEEE Transactions on Instrumentation and Measurement, vol. 56, no. 4, pp. 1181$1188,2007$.

[51] C. Estatico, G. Bozza, A. Massa, M. Pastorino, and A. Randazzo, "A two-step iterative inexact-Newton method for electromagnetic imaging of dielectric structures from real data," Inverse Problems, vol. 21, no. 6, supplement, pp. S81-S94, 2005.

[52] G. Bozza and M. Pastorino, "An inexact Newton-based approach to microwave imaging within the contrast source formulation," IEEE Transactions on Antennas and Propagation, vol. 57, no. 4, pp. 1122-1132, 2009.

[53] H. M. Jol, Ground Penetrating Radar: Theory and Applications, Elsevier Science, Oxford, UK, 2009.

[54] J. H. Richmond, "Scattering by a dielectric cylinder of arbitrary cross shape," IEEE Transactions on Antennas and Propagation, vol. 13, pp. 334-341, 1965.

[55] M. Bertero and P. Boccacci, Introduction to Inverse Problems in Imaging, IOP, Bristol, UK, 1998.

[56] G. Bozza, C. Estatico, M. Pastorino, and A. Randazzo, "A numerical assessment of the semiconvergence behavior in an inverse-scattering approach to electromagnetic imaging," in Proceedings of the IEEE Instrumentation and Measurement Technology Synergy of Science and Technology in Instrumentation and Measurement (IMTC '07), Warsaw, Poland, May 2007. 

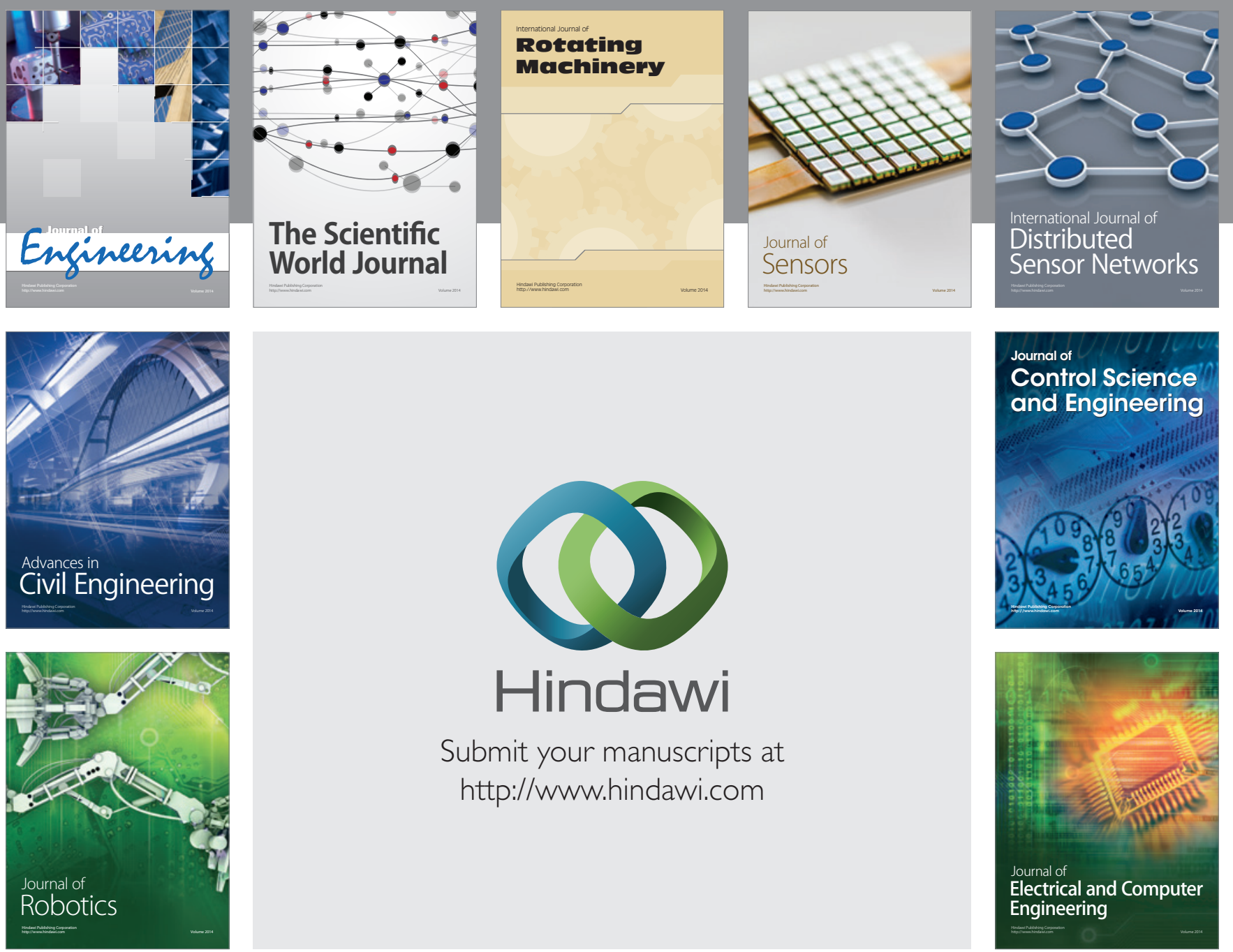

Submit your manuscripts at

http://www.hindawi.com
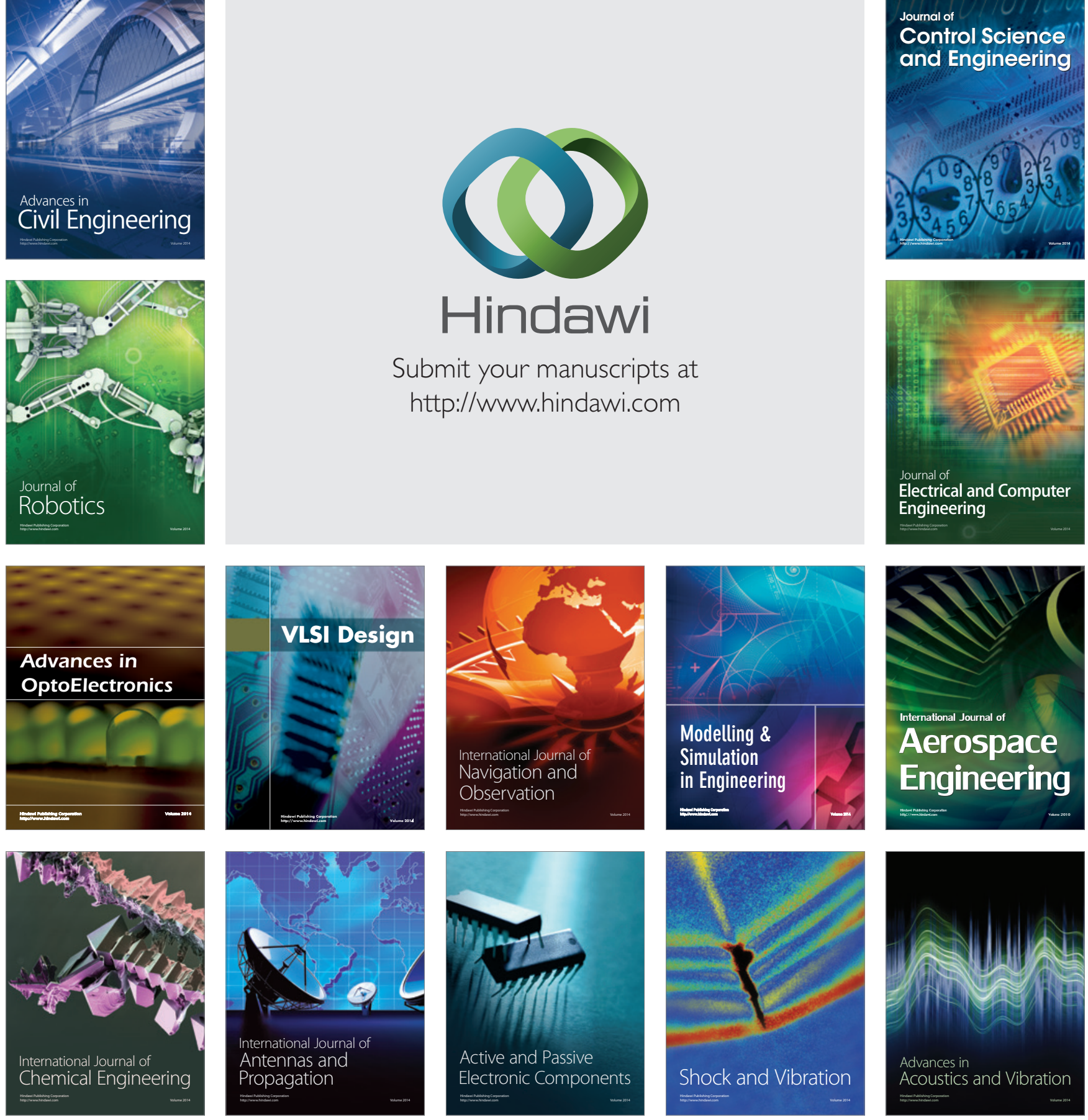